\title{
1 The Digital Hug: Love and Care from a Distance
}

We need to remember the "press" in an impression. It allows us to associate the experience of having an emotion with the very affect of one surface upon another, an affect that leaves its mark or trace. So not only do I have an impression of others, but they also leave me with an impression. ${ }^{25}$

I look into my dad's ear. The image is grainy and pixelated on my phone. Speaking loudly, I try to explain that he should hold the phone in front of him so I can see his face. "What is grandpa doing?" my daughter chimes in. He finally manages to hold the screen somewhat steady in front of him. The lighting in his room at the nursing home is not adjusted for online streaming, and my dad seems unfamiliar with the best influencer angles from which to film oneself, so the image looks grainy, and his face remains in shadow. But at least we can see his silhouette, and he responds in the affirmative when asked if he can see us. I ask about his day, and he tells me in a hushed voice that he went for a walk in the churchyard next to the nursing home, where he met up with his girlfriend of twelve years, who still lives in her own apartment a couple of blocks down the road: "We sat at either end of a bench to keep a distance. No touching, I told her. But it was nice seeing her. I had thought perhaps I was never going to do that again.” (KV)

Although we may sense the outline of a pen if it is placed on our palm, we get a different sensation of its shape, texture, and weight if we move our hand, grasp the pen between our fingers, run our fingertips along its edges. Haptic sensing differs from other senses such as seeing and hearing in that it is bidirectional, meaning that it is dependent on movement. Haptic sensing involves not only tactile cues from the stimulation of sensors in the skin that signal contact with an object (pen on palm), but also kinesthetic information from sensors in muscles, tendons, and joints that send signals about the movement of our limbs and the force used (grasping, weighing the pen). ${ }^{26}$ So when Sara Ahmed in the epigraph above evokes "the press in an impression" as she reflects on the affective impact of emotions, it is notable that the imagery she uses comes from haptic experience: the movement of something being pressed against something else, with a degree of force that leaves an imprint. Significantly, for Ahmed, "others do not have to be nearby to make or leave an impression." ${ }^{27}$

This spatial imagery evokes something pushing outward in the world, and other material retracting, containing. Movement is the key, whether spatially or affectively. In her book on haptics, Lynette Jones - a researcher at the Massachusetts Institute of Technology's department of mechanical engineering, whose work develops tactile, haptic, and thermal displays - reminds us that "in the act of exploring an object we may even change its properties, such as when 


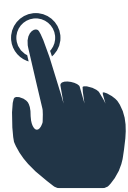

SINGLE TAP

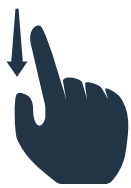

SWIPE DOWN

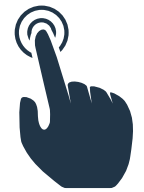

DOUBLE TAP

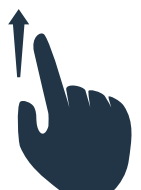

SWIPE UP

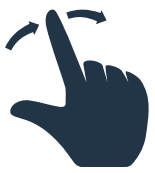

ROTATE RIGHT

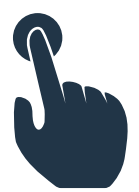

PRESS

AND HOLD

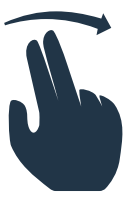

2 FINGER SWIPE RIGHT

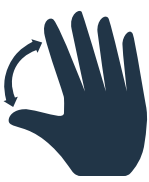

ZOOM IN

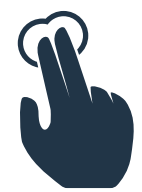

2 FINGER TAP

2 FINGER

DOUBLE TAP

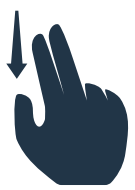

2 FINGER SWIPE DOWN

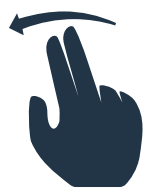

2 FINGER SWIPE LEFT

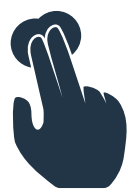

2 FINGER

PRESS

AND HOLD

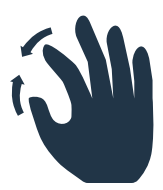

ZOOM OUT

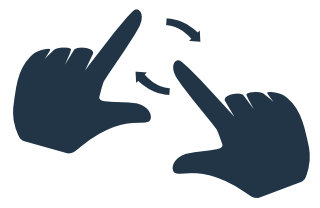

ROTATE

Figure 1: Graphic symbols/icons/pictograms describing digital gestures illustrating the way we touch screens. Image Credit: Macrovector/Shutterstock.com 
we exert too much force on a ripe strawberry and crush it, permanently altering its shape." ${ }^{28}$ Touching and being touched, in the sense with which we are concerned in this book, is about reaching out and reacting to that which pushes itself against us. It is a double movement that binds entities together and has potential to alter those involved: leaving marks or traces, or furthering a healing process ${ }^{29}$ in either case changing the state of that with which we engage. It is about more than one surface interfacing with another, in the same sense in which haptic sensing is about more than tactile cues - skin on skin, skin on screen - but involves the whole situated moving body (muscles, tendons, joints, and all) conditioned by geographical, intersectional structures and a particular emotional and psychological orientation. Touch releases the hormone oxytocin, which is believed to be related to trust and social bonding. ${ }^{30}$ Yet the way touch is experienced is conditioned by things such as temperature, force, and velocity, ${ }^{31}$ as well as by our previous experiences, and by the person who is touching us. ${ }^{32}$ Indeed, studies show that humans can detect emotions such as anger, fear, disgust, love, gratitude, and sympathy when a stranger touches them on the skin of their arm, even if they do not see the touch. ${ }^{33}$ Moreover, people react differently to human touch and machine touch. ${ }^{34}$

Touch can result in tactile and kinesthetic as well as emotional stimuli. So what happens when, in the time of corona, physical touch becomes potentially contagious and therefore needs to be limited? Certainly, many people touch surfaces less; they touch each other less. Yet we find ways to touch and be touched, to move others and create movement within ourselves, despite our isolation and the reduction in our number of contacts with other people. It can be argued that the pandemic hit at a technological point when our ability to reach others without physically engaging with them was unprecedented. The pandemic has shown just how much social life is able to continue without our moving away from the screen to go to other places. However, it has also made visible the painful rifts between those who are sufficiently privileged to be able to stay at home during lockdown and those who have to face the virus as they work in hospitals, clean potentially infected surfaces across the city, keep supply routes open, or deliver the goods bought online by fearful or bored citizens whose main interlocutor is digital media. Who has time to post about the virus and the tedium of quarantine on Facebook? Who has less time to explore online social gatherings because they are multitasking, simultaneously "working from home" and taking care of children or elderly family members? Who is at increased risk of loneliness and isolation? What opportunities do we have to forge new intimate relationships - with lovers and friends - and how is the love that 

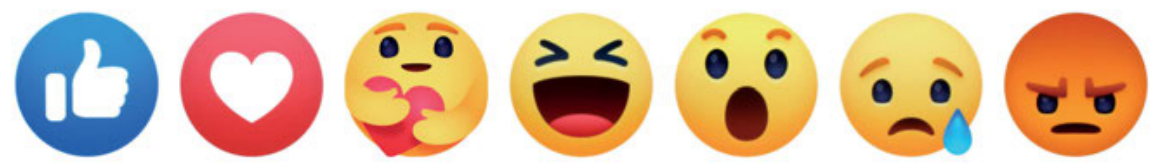

Figure 2: In April 2020 Facebook added the care reaction emoji, giving Facebook users the option to react to posts with a digital hug. Image Credit: rvlsoft / Shutterstock.com 
knits together families and communities affected when people are unable to travel or convene in public spaces, and when self-isolation is recommended for many groups? As for our booming digital lives, they take part in turning the last forty years of work in the field of human-computer interaction into critical everyday experiences and experiments.

As the first lockdowns wore on or were reinstated (and indeed, whenever a lockdown is lifted and the prospect of a new one surfaces), the initial relief that surprisingly many things can be dealt with remotely turned into a sensation of the limits of interacting with others on online platforms. It was as if the digital amplified the longing for the tactile. This seemed particularly acute when the need arose to comfort, console, or share grief. As artist and cultural theorist Emily Rosamond notes in a lecture from 2015 on digital intimacy, tangible space, and the experience of losing a close family member: "In the face of all this - in the face of death - a digital hug has no currency. It is not adequate to the weight of the burden which must be shared." ${ }^{35}$ For Rosamond, a digital hug is somehow too light; there is not enough pressure on the skin to make an impression. When she lost a close family member, she therefore chose to travel in order to be present at the funeral. This opportunity was not available during the COVID-19 lockdowns, which meant that in the face of death, anxiety, and stress, people had to find ways of making a digital hug suffice. Facebook visualized the hug with its "care" emoji, introduced in April 2020 to enable people to show support during the crisis. Amid the ultra-short movement contained in a reaction emoji, a smiley clasps its small yellow arms tightly around a red heart. This is the first Facebook reaction emoji that is not just a facial expression or a symbol: it gives the smiley bodily features, and gestures toward a haptic experience that involves movement of the arms, and a body touching someone else's heart. The digital hug is the key scenario in this first chapter, which is about the ways - and costs - of making a digital hug suffice and creating intimacy at a distance. That is, this chapter concerns the limitations of and opportunities for expressing the caring emotional directionality toward others that characterizes close relationships when physical encounters are restricted and play out digitally. Centering on touch as movement and the mapping of interaction, we look at the use of dating apps and mental health apps during spring 2020, as well as the introduction of contact tracing apps - a kind of reverse dating app - to chart the temporal and spatial structures of intimacy and the points of contact among people, and between people and technology. 


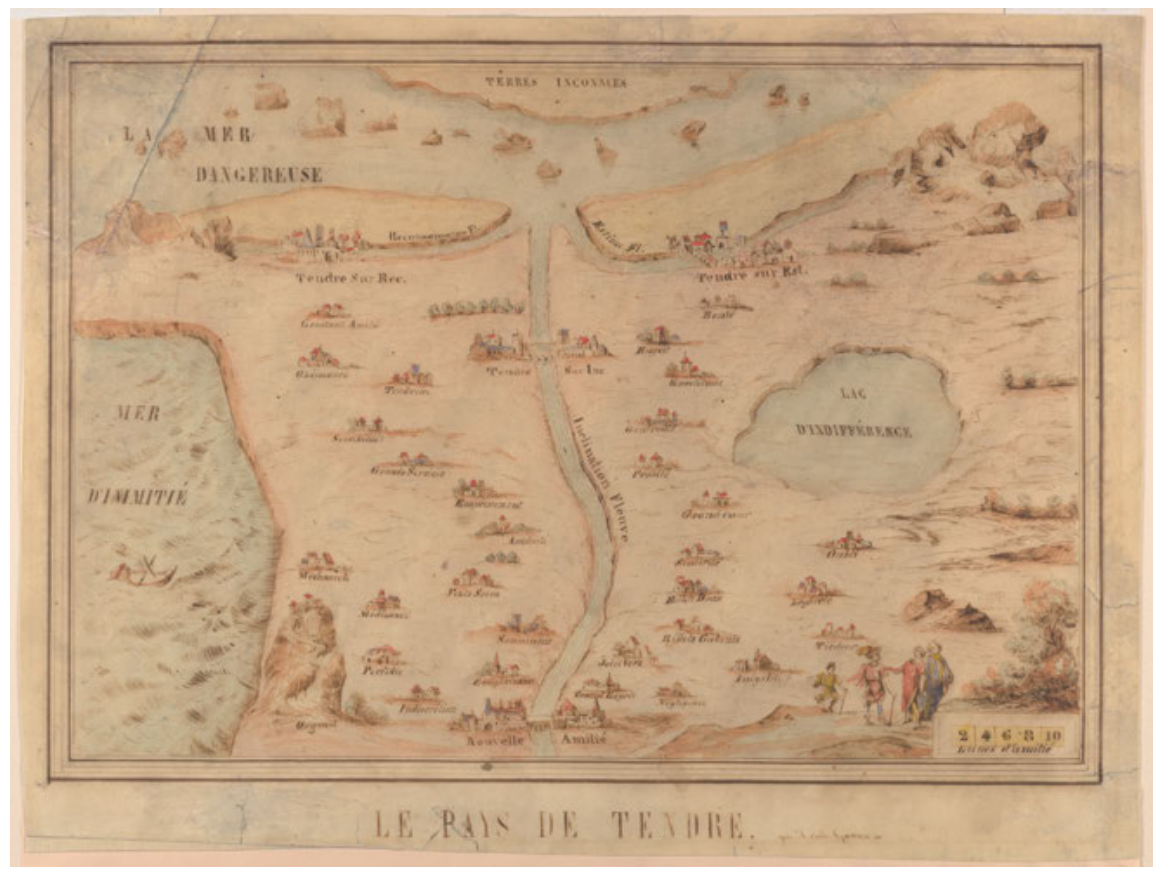

Figure 3: "Le Pays de Tendre" (1800), originally created by Madeleine de Scudéry in 16531654. Image Credit: Cornell University - PJ Mode Collection of Persuasive Cartography 
To understand the intermingling of emotions, bodies, and screens in light of the different forms of social distancing brought about by the pandemic, it is worth taking a step back and situating our immediate experience in a larger culturalhistorical trajectory. A noteworthy early rendition of the relationship between emotion and movement is the famous seventeenth-century Carte de Tendre, which mediates the experience of intimacy and love as a journey by imagining affective states on a geographical map. It is believed that the Carte de Tendre was conceived in Paris during the winter of $1653-1654$ as a social game and collective project among the female intellectual circle of society hostess Catherine de Vivonne, marquise de Rambouillet. ${ }^{36}$ The map was subsequently integrated as an engraving (attributed to François Chauveau) into the novel Clélie (1654-1661) by Madeleine de Scudéry, a writer of romances who was part of Vivonne's circle. Satirized by Molière in Les Précieuses ridicules (1659), this circle was a literary salon of educated women for whom love and courtship were cherished objects of attention and debate. However, it has since been argued that the map can also be read as a riposte to the seventeenth-century military cartography of French scientists and engineers. In this reading, the Carte de Tendre as a collective feminist project reconfigures the political into the intimate personal realm, exploring the nuances of relationships not only between men and women, but also between king and subject, authority and subservience, freedom and obligation. ${ }^{37}$

The Carte de Tendre charts the possible paths from new encounters (represented by the city of Nouvelle Amitié, "new friendship") via the River of Inclination, which flows directly toward the city Tendre-sur-Inclination, but is surrounded by perils such as the flat Lac d'Indifférence ("lake of indifference"), La Mer Dangereuse ("the dangerous sea"), which is full of cliffs, and the unruly waters of Mer d'Inimitié ("the sea of enmity"), where a ship is struggling to keep afloat. Small villages adorn the route on land, designating flirtation techniques such as the Billet Doux and Billet Galant ("love notes"), or emotive states such as Empressement ("eagerness") or Complaisance ("desire to please"). The route on land may also lead to the two larger riverside cities of Reconnaissance ("recognition") and Estime (“esteem"), modes of tenderness reached by more laborious paths. The map is thus a complex charting of an emotional landscape where affective sensibilities take geographical and infrastructural form. As visual arts scholar Giuliana Bruno aptly notes, "the Carte de Tendre makes a world of affects visible to us. In its design, grown out of an amorous journey, the 


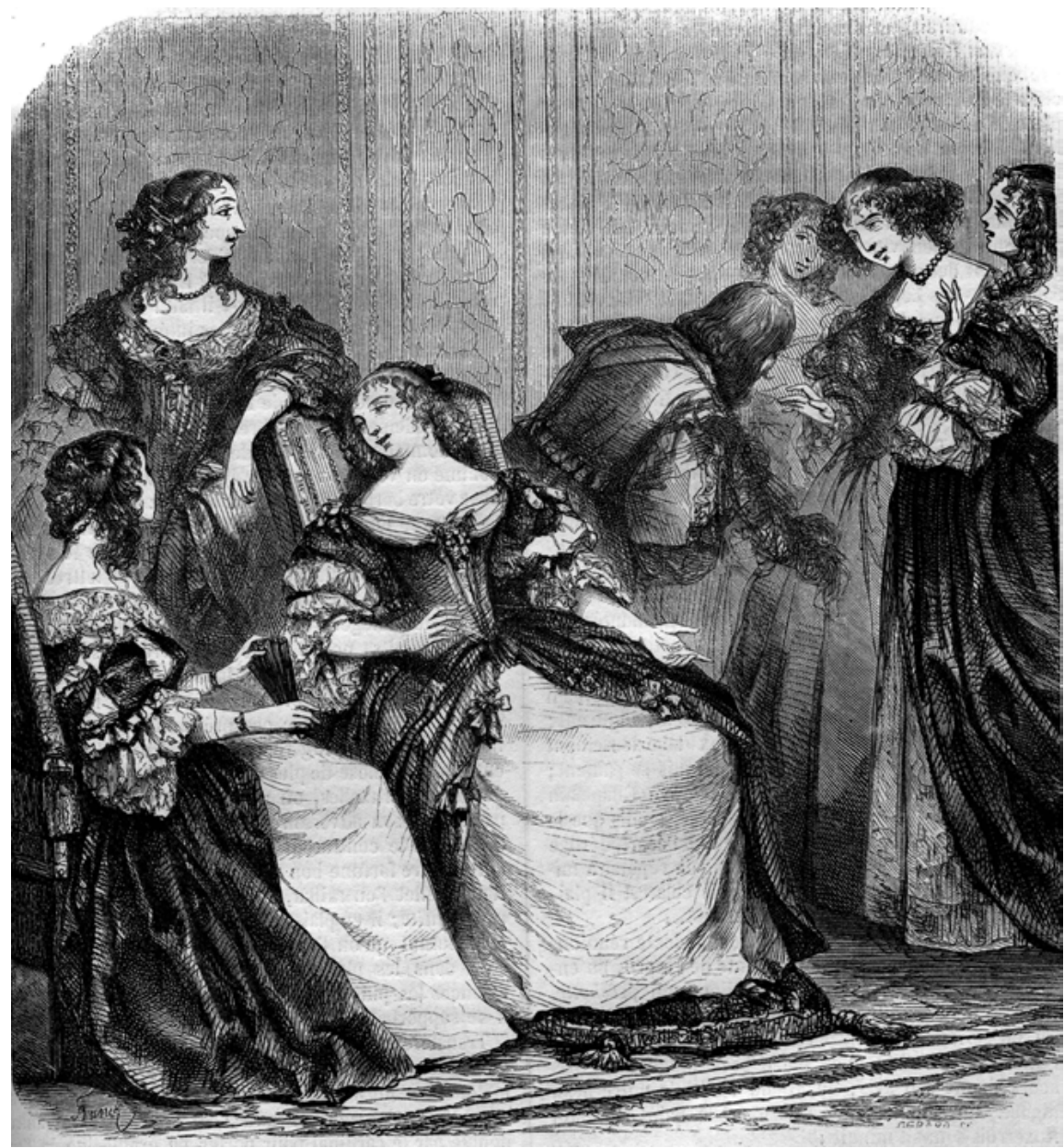

Figure 4: Rendering of Catherine de Vivonne, Marquise de Rambouillet. France, nineteenth century. Image Credit @ 2021. White Images/Scala, Florence 
exterior world conveys an interior landscape. Emotion materializes as a moving topography. To traverse that land is to visit the ebb and flow of a personal and yet social psychogeography." ${ }_{38}$ We can speculate about how a map that took account of the pandemic's regulation of touching and being touched would look. Would it be a dating app, or a contact tracing app? Would it encourage intimacy or distance? Love and courtship might have changed considerably since the seventeenth century, but we are no less occupied by the attempt to chart our emotions; we even automate our ability to register them through emotion detection technology. As a projection screen for the personal yet social, the Carte de Tendre is an apt reminder to think about the interfaces through which we engage with lovers and loved ones during quarantine, curfew, or lockdown. Historical maps such as the Carte de Tendre, understood as "exemplary tools in the articulation of new attitudes, exposing controversial states of social awareness," ${ }^{39}$ remind us to look for the political implications embedded in the everyday technologies that help to reconfigure our intimate sphere today - the social sorting and intrinsic biases that are part of the configuration of digital intimacy. Maps today are predominantly digital and interactive; they chart our movements, and they predict where we want to go. Geolocation features on dating apps facilitate online conversations or physical meetings. Most of these devices chart our emotions in some way - less as a moving topography captured through the art of mapmaking, and more in the language of metadata. Statistically speaking, how many times do we exchange messages on Tinder before meeting in person? How far are we willing to travel to meet a potential lover? The experience of communicating through a screen becomes a dynamic metadata map, ripe for data analysis.

If we look at the statistics regarding online dating during the first round of COVID-19 lockdowns in early 2020 (which started and ended on different dates in different countries), it is notable that many dating services reported an increase in the length of conversations, even though Match Group's (the company behind Tinder and OkCupid) stocks tumbled twenty-five percent when the prospects looked gloomy for products designed to facilitate physical meetings. Yet, despite these dire conditions, Tinder reported that its users worldwide made three million swipes on Sunday, March 29, 2020, the greatest number of swipes the app had ever registered in a single day. In the UK, daily conversations rose by twelve percent between mid-February and the end of March; in the US, conversations during the pandemic were twenty-five percent longer than normal. 
Inner Circle reported that the number of messages being sent increased by 116 percent, and OkCupid noted a twenty percent rise in conversations, while Hornet saw a thirty percent increase in social feed engagement. ${ }^{40}$ There was also an increase in video dates ${ }^{41}$ : Hinge rolled out a "Date from Home" option that allowed users to start a video chat; Plenty of Fish introduced the opportunity to livestream with potential matches; Bumble, which had offered video calls as an option since 2019, saw a ninety-three percent rise in video calling during the week after the then US President Donald Trump declared a national emergency. ${ }^{42}$ In the second week of April 2020, Match.com surveyed its users about changed habits during the pandemic: while only six percent had used video chats before COVID-19, now sixty-nine percent responded that they were open to video chatting. ${ }^{43}$ Communication studies scholar Stefanie Duguay has noted that platforms such as Her and Lex encouraged acts of care and self-care by promoting safety. ${ }^{44}$ When meeting strangers physically becomes fraught with fear, longer online conversations, with or without images, seem an obvious choice. In the New York Times, Helen Fisher, Match.com's chief scientific adviser, called for "slow love," arguing for the benefits of people getting to know each other online before meeting in person, and thus turning what might have appeared to be a threat to the dating industry into an opportunity to transform the culture of fast, fleeting love that the industry is often accused of stimulating. ${ }^{45}$

Temporally, then, the journey along the River of Inclination depicted on the Carte de Tendre may have become longer and slower during lockdown and quarantine; but the ability to make a digital hug suffice becomes even more pertinent. How to trigger the release of oxytocin when no human touch is involved? How to move yourself under lockdown? Maps on dating apps that display geolocation are normally a matching criterion as well as a warranting feature that serves to reassure users that there is a real physical person behind a given online profile. ${ }^{46}$ Yet these maps' function of linking physical bodies to digital profiles relates to practices that have now become constrained due to the pandemic. It simply seems less relevant whether the person you are talking to is ten miles or a hundred miles away. Some platforms responded by making features that are normally subscriber-only free to everyone. For example, Tinder's "passport" allows users to match and chat to people in different locations than their own. It works somewhat like a virtual trip, insofar as it allows you to select a new location and interact with matches in your new (virtual) vicinity; during spring 2020, online dating with people in remote places provided a rare opportunity to "travel." Happn, the app that matches people based on whether their paths have crossed in real life, extended the radius so that users could match 
with people within 120 kilometers. Such changes may explain why many dating apps saw increased matching and messaging but less willingness to pay for subscriber features while countries were under lockdown, although the latter increased again as lockdowns lifted. ${ }^{47}$ Indeed, as film and digital media scholar Diego Semerene argues: "So much cruising, so little sex: from the question, 'What do you want to do to me?' as a strategy to fish for words that, unlike the body, can hit the fantasy at its heart, to the exposition of exhaustive scenarios of how the sexual encounter should take place even though, or precisely because, it never will." ${ }^{\prime 8}$ Because the physical encounter is prohibited, the touch has to be conjured from the words that can be exchanged.

What is interesting here in relation to the interlinking of touch, movement, and intimacy is that although many dating platforms do not seem to have increased their user numbers during lockdowns (unlike online meeting platforms such as Zoom), there has been a change in how those who already had the apps use them during the pandemic: users engage in longer conversations online, rather than short interactions, because moving and touching now have to take place on the platform. The map of tender of the pandemic is thus one that is measured temporally and charted in metadata, rather than manifesting emotion as topography as in the Carte de Tendre. What we see in the statistics about the use of dating apps is not motion through a landscape, but an exteriorization of the development of emotions, measured in time spent interacting with someone. Notably, in this process the need for knowing that we are interacting with another human and not a chatbot seemingly becomes less important. Indeed, digital technologies may be better equipped to perform digital hugs than humans.

Some of the apps that did increase their user numbers push the limits of what a digital hug can mean, insofar as the interlocutor is precisely what many dating app users normally fear: chatbots. While this was already a growing market before lockdown, the worldwide quarantines increased the popularity of artificial-intelligence-driven chatbots that use natural language processing and machine learning to have conversations with users and offer emotional support. The mental health app Wysa saw a seventy-seven percent increase in new users during February and March. ${ }^{49}$ Wysa is the first artificial intelligence mental health app to meet the clinical safety standards of the UK's National Health Service, and it was recommended by the health app evaluation and advice organization ORCHA as the best app for COVID-19 stress and anxiety. The app 
relies on techniques such as cognitive behavioral therapy, meditation, and motivational interviewing, but it also offers the opportunity to contact real-life mental health professionals (for a fee). It was developed as part of a project to get machine learning models to help with the detection of depression. This project found it difficult to persuade people to accept help from a therapist, even when depression was detected, but the chatbot feature became surprisingly popular. ${ }^{50}$ Wysa is careful to stress that it is not meant to replace human therapists. ${ }^{51}$ Nevertheless, apps such as these seem to respond to the need to share your thoughts and have them reflected back to you, and they provide a format where this is possible without your having to sit opposite a therapist. However, with these apps we also tap into a growing research field that emphasizes the intersectional biases in machine learning, ${ }^{52}$ and this reminds us that just as we (unlike Molière) should not write off the Carte de Tendre as the product of a group of privileged, educated women with nothing better to do than muse about love and flirtation, so dating apps and mental well-being apps are far from innocent and benign, and should be accorded serious scholarly attention.

In her reflections on digital intimacy and the impetus to share as something that has transactional value, Rosamond compares dating apps to an older technology: eighteenth-century British literature. It has been argued that this literature developed characters with complex interior lives in response to the introduction of overseas commodities, which challenged people to make items from far beyond their horizons into private possessions, meaning in turn that interiority became a form of commerce. Rosamond tentatively argues: "Perhaps I could suggest that online dating avatars are like literary characters, producing commerce of shared feeling and/or shared interests between readers and characters - except that all of the readers are also characters, and all of the characters are also readers." ${ }^{{ }_{33}}$ Many chatbots seem indeed to operate through this logic, facilitating a feeling that we - by sharing our vulnerabilities with the persona cocreate a character that we in turn commission to listen to us.

A very popular chatbot is Microsoft's XiaoIce, a virtual teenager that ranks among China's celebrities. ${ }^{54}$ XiaoIce was launched in China in 2014, and had 660 million active users in 2019.55 Li Zhou, Jianfeng Gao, Di Li, and HeungYeung Shum from Microsoft Research have charted how a user's emotional connection with Xiaolce builds over a two-month period: from exploring her features and functions, asking questions such as "Are you human?" and "What functions do you have?" in the first session; talking to XiaoIce about hobbies 
and interests in session six, after two weeks; starting to treat the bot as a friend and bringing in the user's personal life after four weeks, in session twenty; talking to the bot almost every day after seven weeks, around session forty-two. After two further weeks, around session seventy-one, Xiaolce is the user's preferred choice when they want to talk to someone. ${ }^{56}$ These bots' ability to establish intimate bonds with individual users reveals that technology can provide emotional support by being much more available than any "real-life" friend would be. In these cases, a digital hug carried out by technology seems to suffice, and sometimes even to exceed a physical hug, because it is so much more readily available. We see here how the mapping at work in the interaction with XiaoIce plays out in a temporal register: it is the duration and intensity of the interval in the interaction that administers the press in the impression invoked by these apps. A sense of proximity arises from an increased ability to interact and share. At the same time as health authorities around the world identify physical proximity as a risk factor in the spread of coronavirus, particularly over time, we find other ways of moving ourselves emotionally and being in proximity with others - human or nonhuman.

During the pandemic, a new range of apps also arrived: the contact tracing and health code apps that prescribe who can move around and who needs to stay in quarantine. These apps map and administer distance and intimacy alike, often with duration of proximity as the key factor. In many countries they have become deeply ingrained as a way of managing and controlling movements and relationships.

Not surprisingly, the public debate and rhetoric around the use of these apps to manage both the population and the disease mimic existing discourses, debates, and sentiments about technology and tracking in various regions of the world. Reports about China's health code app quickly sparked privacy concerns, with the New York Times calling it "a troubling precedent for automated social control." ${ }^{57}$ China introduced the app in response to the reopening of cities in February 2020, and the codes were added to the mobile payment platforms Alipay (partly owned by the tech giant Alibaba) and WeChat (owned by another tech giant, Tencent). ${ }^{58}$ The codes function somewhat like health passports, determining your ability to travel and enter certain places. Although the app is individually adapted in different Chinese provinces, a green QR code generally means you can move around with no restrictions, while a yellow code designates some form of home isolation, and a red code means quarantine. The latter 


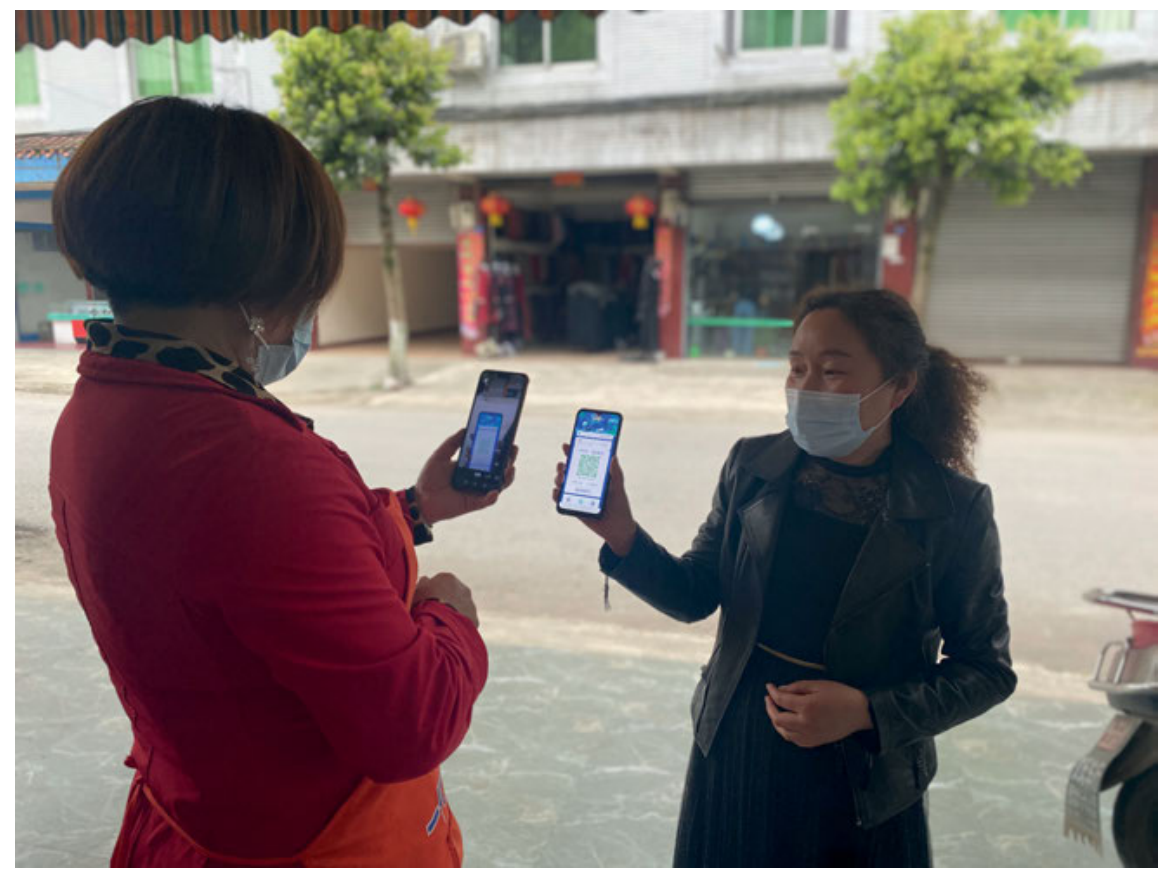

Figure 5: The Chinese health code app initiated at the beginning of the pandemic and criticized (for instance, in the New York Times) for its automation of social control. As shown on this picture, the app works through a QR code that can be scanned by another person, e.g. on a smartphone. A year later, Denmark launched its own "corona passport," restricting access to restaurants, museums, etc. Image Credit: Private Photo / Ting Huang 
two codes are assigned to people who have been in contact with an infected person, visited an area with many infected people, or reported symptoms. The New York Times reported that each time a code is scanned, the location is sent to the system's servers, enabling the tracking of movement over time. ${ }^{59}$

The exact criteria according to which the codes are assigned remain opaque, generating critique from users who feel their color code is arbitrary. Nonetheless, the codes are used to guide movement through cities and prohibit contact with others - quickly becoming an entry pass to public transit as well as hotels, restaurants, and shops.

Apart from privacy concerns, and the fear that the code system may remain in place and be integrated into future smart city developments, there has also been significant concern because the elderly, who are particularly vulnerable in terms of health, are less likely as a socioeconomic group to own or be able to operate a smartphone. ${ }^{60}$ The usefulness of these apps - not unlike dating apps is dependent on the number of users who install it, which in turn is dependent on political structures and popular sentiment. In India, the app Aarogya Setu (meaning "the bridge for liberation from the disease") had more than fifty million installations in the first thirteen days, overtaking Pokémon Go to become the world's fastest-growing mobile app. ${ }^{61}$ After four weeks it was made mandatory for all workers in the public as well as private sectors, as is the use of similar apps in Indonesia, Vietnam, and Qatar. ${ }^{62}$ Other countries, where the use of such apps is still voluntary, struggle to get citizens to participate, due partly to privacy concerns and partly to perceptions of the apps' effectiveness. The role of the multinational tech giants in the rollout of app-based solutions to disease control deserves a whole study of its own: the topic speaks to the geopolitical negotiation of movement in a global pandemic that nation-states are seeking to alleviate. While most apps are designed nationally and developed as part of disease control strategies within the boundaries of the nation-state, a key decision has to be made regarding whether the data architecture should be centralized - in which case the data is uploaded to a server controlled by the government health authority - or decentralized, in which case it is stored locally on users' devices. Significantly, Apple and Google partnered up in spring 2020 to enable a decentralized approach, which came to be seen as more secure and less problematic in terms of privacy. While it may at first sound paradoxical that big tech is better attuned to privacy concerns than governments, various scandals have taught these companies how dependent they are on users adopting the technology. The companies' more economically motivated approach may therefore focus on mitigating users' concerns in order not to lose customers. As global rather than nationally available technologies, these apps tap into surveil- 
lance-capitalist modes of thinking about data as a noninvasive resource ripe for extraction. Coupled with disease control, this makes the choice to "opt out" all the more ambivalent: after all, who does not want to help stop the pandemic? At the same time, the door is also left open to a host of uncertainties pertaining to the risk of false positives (for instance, the app cannot see if there are barriers in place that prohibit contamination) or false negatives (for instance, unlike GPS, Bluetooth cannot detect that you have entered a space that an infected person just left) and thus to the usefulness of these apps for anything other than data collection.

However, these apps arguably perform a form of affective mapping that is akin to the Carte de Tendre, except that they are less focused on developing amorous feelings and more on managing the fear of being contaminated by others. Contact tracing apps emphasize geography in a different way than the dating apps and mental health apps on which, according to behavioral statistics, people spend more time talking to each other during lockdowns. Temporality is also a key marker in the mapping performed by contact tracing apps insofar as the duration of an interaction is essential for the estimation of risk. Moreover, these apps use physical proximity as a matching criterion, in a similar way to dating apps' use of geolocation, although here the "dating" takes place between anonymized phone IDs rather than appealingly filtered profile photos. In Europe, various contact tracing apps have been modeled on forms of partner notification that have been used (in the UK, for instance) to anonymously inform individuals that they have been named as a previous sexual partner by someone who has contracted a sexually transmitted infection, and that they should therefore get tested. However, while we can assume that most people usually have some idea of who they have had sex with and can narrow it down to a fixed list whose phone numbers can be found, the ephemeral nature of the touch of coronavirus means that we are far more incalculable in terms of spreading the disease, and much less likely to know to whom we might have passed it on. The solution in many of the apps - and the technology that Apple and Google joined forces to implement - exploits what Wendy Chun has identified as the "chatty" and "promiscuous" ${ }^{63}$ qualities of networked devices by using Bluetooth to log other phones that come within a certain distance. These apps aim to ease privacy concerns by storing the information locally on the individual device rather than in a central database, and by not saving location data. The decentralized infrastructure means that health authorities do not have the option to perform big data analyses and map outbreaks geographically. Yet for the individual phones, the identification of geographical proximity is essential to create an "event" that is stored in the 
system. A constant negotiation of individual and collective experiences of people and devices, nation-states and multinational companies, is thus at the core of the contact tracing app as a cultural technique for disease management insofar as the app is simultaneously both deeply personal (hence the host of privacy concerns) and fundamentally supra-individual. ${ }^{64}$

This blurring of the individual and societal body is also recognizable in questions concerning immunization. Of course, the question of whether immunity can emerge in the population is dependent upon whether individuals' immune systems have responded to the virus after contracting the disease or being vaccinated: it pertains to concrete bodies that have either been touched by pathogens or received an appropriate vaccine dose. Immunity in this sense lowers the individual's risk of contracting the disease or becoming fatally ill, but it also means that the individual takes part in a collective effort to achieve herd immunity, thereby lowering the general risk of infection. Thus, immunization is almost a tradable good, embodied in altercations about access to sufficient vaccines between nation-states, institutions, and interest groups, as well as in stories of people who consciously seek exposure to the virus, e.g. at COVID parties or the like. ${ }^{65}$ This is also reflected in "corona passports": any sign that one's body cannot pass on the disease - whether demonstrated by a negative test result, or by antibodies from one's having previously contracted COVID or received a vaccine - can become a gateway across national borders, or simply to access to restaurants, cafes, hairdressers, etc.

Indeed, the experience of being part of a gigantic invisible network is palpable when you download one of these apps. For instance, the interface of the Danish app Smittestop (“contamination stop”) shows a green pulsating circle. It notifies you if you have spent more than fifteen minutes within one meter of someone who has subsequently turned out to be COVID-19 positive. There is no swiping or interaction involved - it works invisibly on its own, in the background - and unlike most other apps, you receive no dopamine reward from notifications. The only way to interact with the app is to press the green circle to stop the monitoring, in which case the circle turns red. The feeling of security it provides thus operates not at the individual but at the collective level: it gives the individual the sensation of playing a role in a crisis that otherwise offers very little room for individuals to contribute on a larger scale and make an impression. The digital hug here is the light touching of two UHF radio shortwaves sent from two different devices, registering and logging each other's presence in local databases, creating vast personal social networks that (unlike the social media we sign up to) are not meant to be seen and traced back to specific 


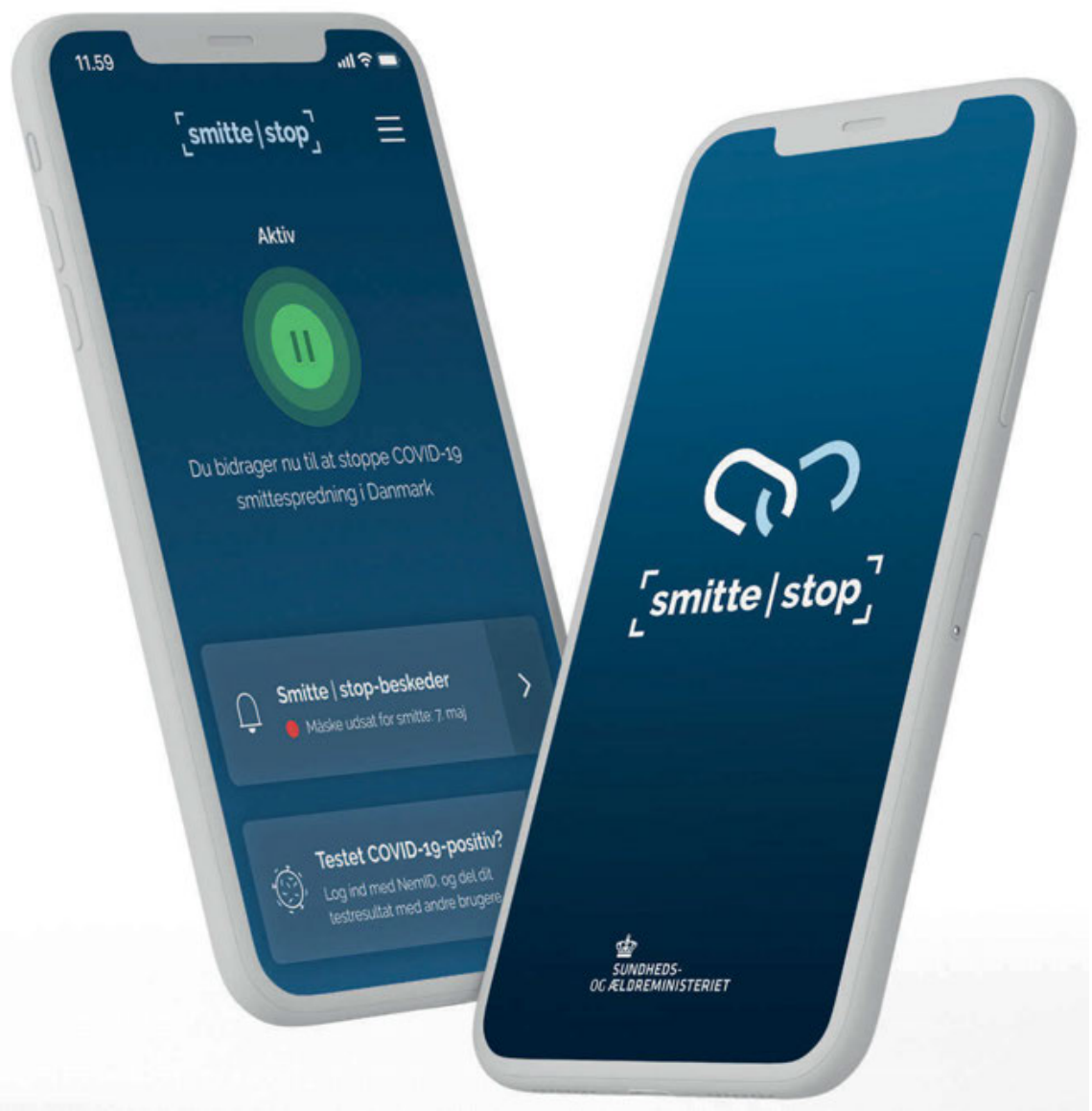

Figure 6: The interface of the Danish app Smittestop. Image Credit: The Danish Ministry of Health 
geographies. It seems like a radical version of the Situationists' psychogeographical practice of liberating the urban fabric by creating alternative mappings. Paradoxically, the invisibility of these mappings makes them all the more powerful. They remain notably uninterested in psychology or the idea that individuals and technological systems may have intentions or desires; rather, individuals are simply potential carriers of the virus in a massive digitally linked network. The need for a digital hug has thus been transformed into a gigantic, chatty and digitally promiscuous societal squeeze. 


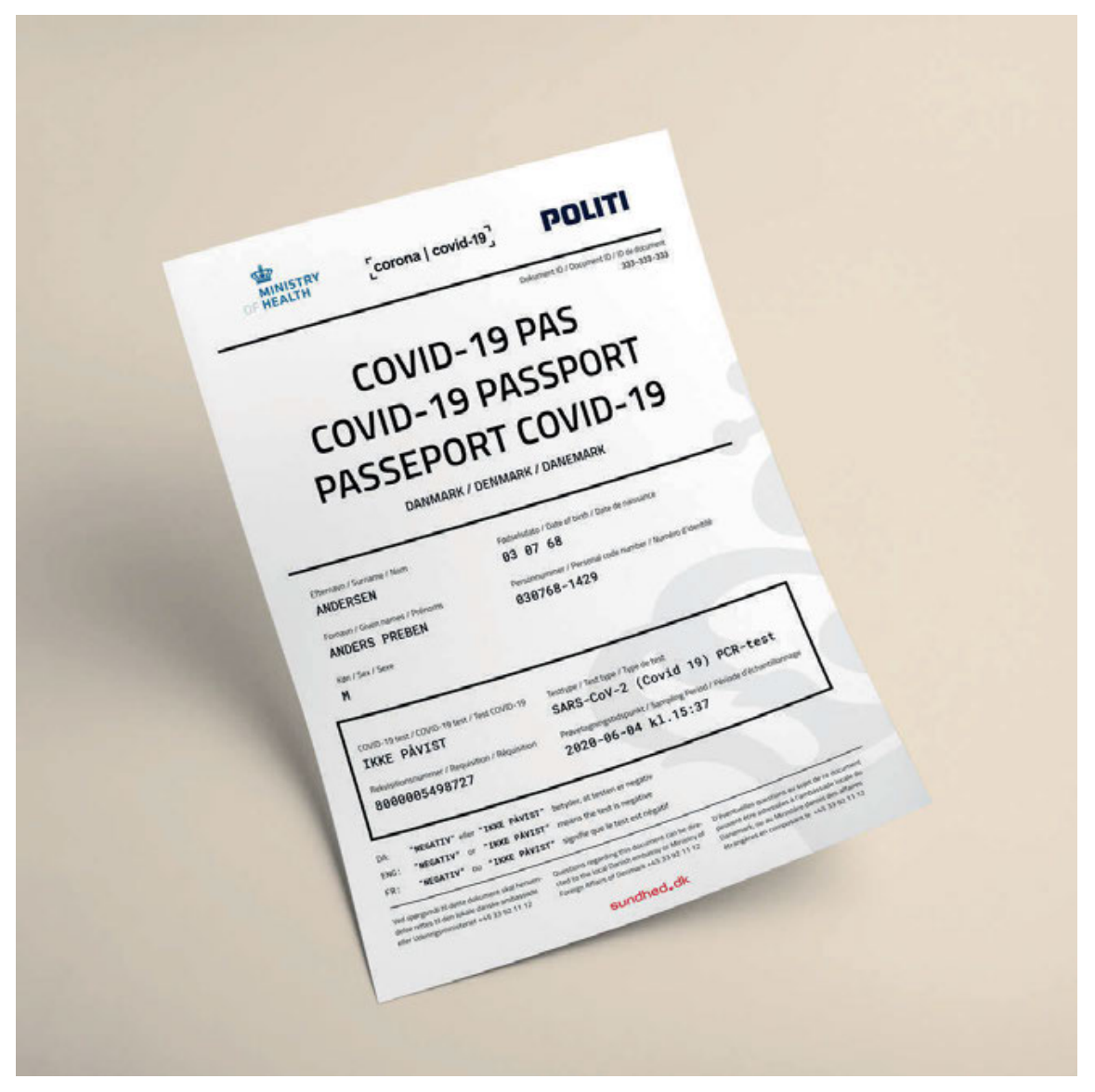

Figure 7: Example of a Danish corona passport. Image Credit: Sundhed.dk 